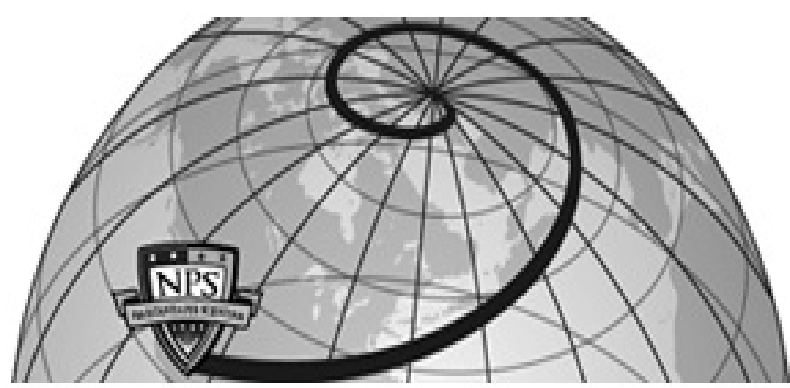

Calhoun: The NPS Institutional Archive DSpace Repository

\title{
Optimal Nonlinear Feedback Guidance for Reentry Vehicles
}

Bollino, Kevin; Ross, I. Michael; Doman, Dave

The American Institute of Aeronautics and Astronautics (AIAA)

https://hdl.handle.net/10945/29668

This publication is a work of the U.S. Government as defined in Title 17, United

States Code, Section 101. Copyright protection is not available for this work in the United States.

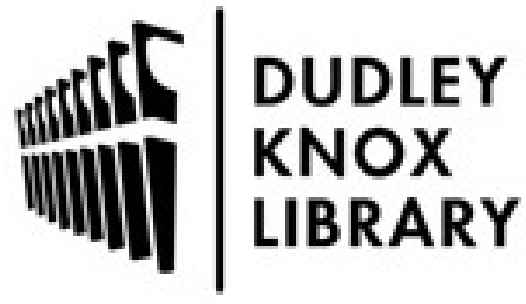

http://www.nps.edu/library
Calhoun is the Naval Postgraduate School's public access digital repository for research materials and institutional publications created by the NPS community. Calhoun is named for Professor of Mathematics Guy K. Calhoun, NPS's first appointed -- and published -- scholarly author.

Dudley Knox Library / Naval Postgraduate School 411 Dyer Road / 1 University Circle Monterey, California USA 93943 


\title{
Optimal Nonlinear Feedback Guidance for Reentry Vehicles
}

\author{
Kevin P. Bollino* and I. Michael Ross ${ }^{\dagger}$ \\ Naval Postgraduate School, Monterey, CA 93943 \\ David D. Doman ${ }^{\ddagger}$ \\ Air Force Research Laboratory, WPAFB, OH 45433-7531
}

\begin{abstract}
It is well-known that onboard, real-time, optimal trajectory generation for reusable launch vehicles can substantially increase safety while reducing cost. Arguably, this is one of the most challenging problems in optimal control theory. In recent years, pseudospectral (PS) methods have shown considerable promise in solving such problems. In this paper, we investigate the utility of a PS guidance algorithm for solving this problem. Based on the $\mathrm{X}$ 33, a generic vehicle model is constructed for analysis. It is shown that the PS guidance algorithm is capable of compensating for large uncertainties and disturbances that include greater than Category 5-level hurricane-force wind gusts (Hurricane Katrina was Cat 4). A single algorithm is used to guide the vehicle from its entry condition to a final approach corridor of about $2000 \mathrm{ft}$ away from the landing site. Despite that much research is still necessary, results so far suggest that PS guidance methods can enhance safety considerably. Computational times for unoptimized codes range from about a second to 10 seconds under a MATLAB environment running on legacy computer hardware. This suggests that realtime trajectory reshaping, and even abort guidance, are well within the reach of modern methods and existing computational capabilities.
\end{abstract}

\section{Introduction}

With ongoing efforts to improve the safety, reliability, and cost of reusable launch vehicle (RLV) systems and operations along with the more recent focus on a US Space Shuttle replacement following its retirement around 2012, there is a new opportunity to implement advanced, yet simpler and more effective guidance and control methods than used in previous launch technologies. A key objective that is sought in this endeavor as well as almost all other sectors of technology is the requirement for more intelligent systems that can operate autonomously without much human interaction. Of course this has the underlying stipulation that they operate safely, more reliably, and more efficiently than ever before. To accomplish this objective for the case of an RLV, the guidance and control during ascent, reentry, or landing needs to perform autonomously, online and in real-time. The RLV intelligent control needs to be capable of adapting to rapidly changing circumstances, handling large external disturbances and large parameter uncertainties, re-generating trajectories (i.e. re-planning to a designated or alternate landing site), reconfiguring its controls in the advent of an unforeseen failure, and then re-allocating the controls. In practice, these challenges are met by a substantial amount of pre-flight planning involving predictions in weather, substantial amount of manpower, potential grounding of scheduled launch and a host of other operational workarounds to technical problems. In taking less computer power than a modern digital wristwatch, the Shuttle entry guidance is a marvelous technical achievement wherein a drag-based tracking technique is used for guidance. ${ }^{1}$

"Ph.D. Candidate, Department of Mechanical and Astronautical Engineering, Guidance, Navigation, and Control Lab, Ph. 831-656-3194, Email: kpbollin@nps.edu, AIAA Member.

${ }^{\dagger}$ Professor, Department of Mechanical and Astronautical Engineering, Ph. 937-656-2074, Email: imross@nps.edu, AIAA Associate Fellow.

${ }^{\ddagger}$ Senior Aerospace Engineer, 2210 Eighth Street, Bldg. 146, Rm. 305, Ph. 937-255-8451, Email: David.Doman@wpafb.af.mil, AIAA Associate Fellow.

1

American Institute for Aeronautics and Astronautics 
With the realization of advances in computational power and numerical algorithms, recent research efforts for solving the reentry problem have focused on real-time, on-line trajectory generation, guidance adaptation, and control reconfiguration. For example, reconfigurable control in the form of an optimal control allocation algorithm has been performed at the Air Force Research Lab for such vehicles as the X-40, X-37, and X-33. ${ }^{2-4}$ Reconfiguration capabilities for RLV guidance and control systems has also been rigorously pursued by such efforts as the Marshall Space Flight Center's (MSFC) Advanced Guidance and Control (AG\&C) Program and the Air Force Research Lab’s (AFRL) Integrated Adaptive Guidance and Control (IAG\&C) Program. More on these programs can be found in Refs. 5 and 6. It is now well established that many of the challenges on entry guidance fall under the purview of optimal control theory. Difficulties in solving optimal control problems have reflected difficulties in solving the entry problem.

Over the last few decades, it has become customary to address the difficulties in entry guidance by way of reduced-order modeling and analysis. ${ }^{7,8}$ In a series of papers, ${ }^{9-11}$ Fahroo et al. showed that reduced-order modeling compromise safety by predicting diminished footprints. That is, a reduced-order model may falsely imply that a preferred landing site was not reachable thereby generating a failure mode that could otherwise have been prevented. Thus, entry guidance algorithms that are not based on optimal control and reasonably high fidelity models compromise safety. This concept was further clarified by Shaffer et al. ${ }^{12}$ who showed that optimal control techniques can also address trajectory reshaping in the presence of actuator failures. It has thus become increasingly clear in recent years that optimal trajectory generation is not a mere luxury but an absolute necessity for safe entry vehicle guidance. Furthermore, it is not sufficient to generate optimal trajectories for low-order models, but for sufficiently high fidelity vehicle and dynamical models. At the present time, it is unclear what qualifies as a sufficiently high fidelity model. For example, Shaffer et al. showed that high-fidelity aero models were quite crucial for proper guidance in the Mach-alpha space whereas Fahroo et al. showed that high-fidelity dynamical models were crucial for footprint maximization. One way to address all these issues is to construct models of increasing fidelity for a new kind of analysis that exploits all the modern tools in optimal control techniques. This is precisely the approach adopted in this paper.

In this paper, we use a standard 3-DOF dynamical model for an entry vehicle in a rotating atmosphere around an inverse-square law gravitational field. In recent years, it has been shown ${ }^{13,14}$ that PS methods are capable of generating real-time trajectories for this dynamical model for the Space Shuttle vehicle model parameters. Based on the possibility of real-time optimal control, a PS feedback theory has been formulated by Ross et al. ${ }^{15,16}$ For entry guidance, this approach updates the guidance laws in the so-called Carathèodory sense, rather than the standard sampled data feedback approach. This type of an update permits a longer computational time that may be exploited to solve high-fidelity trajectory optimization problems. An introduction to this approach is described in Ref. 16. Details of the PS method and the spectral algorithm (packaged into a MATLAB-based software program called DIDO) used to generate the optimal controls are described in Refs. 17-20.

The contributions of this paper are several fold: first, we combine many of the emerging concepts in real-time optimal control to address the difficulties in entry guidance. To this end, we circumvent the traditional problems that arise in entry segmentation (such as the concept of TAEM) and instead embark on using a single algorithm to guide the vehicle from its entry conditions all the way to a neighborhood of the landing site. Thus, given the coordinates of a landing site, the goal of the entry guidance algorithm is to guide the vehicle to a final approach corridor for handover to an autoland program. In principle, it is possible for a PS-based method to perform autoland as well, but for the purpose of limiting the scope of this paper, we do not discuss this aspect of the problem. The vehicle model is based on an analytical curve fit of an X-33 vehicle model. It remains to be determined if our approach works with the full table-look up model; nonetheless, as a first step in this new type of analysis, we choose an analytical curve fit. An important result of this paper is the revelation that under the PS guidance law the simplified X-33-based generic vehicle is capable of guiding itself to a landing site despite the extreme situation of hurricane wind effects that exceed Category 5. 


\section{Modeling}

\section{A. Entry Vehicle Model}

The equations of motion used for this study are the standard 3-DOF model,

$$
\begin{aligned}
\dot{r} & =V \sin \gamma \\
\dot{\mu} & =\frac{V \cos \gamma \cos \xi}{r \cos \lambda} \\
\dot{\lambda} & =\frac{V \cos \gamma \sin \xi}{r} \\
\dot{V} & =-\frac{D}{m}-g \sin \gamma+\Omega^{2} r \cos \lambda(\sin \gamma \cos \lambda-\cos \gamma \sin \lambda \sin \xi) \\
\dot{\gamma}= & \frac{L \cos \sigma}{m V}+\left(\frac{V}{r}-\frac{g}{V}\right) \cos \gamma \\
& \quad+2 \Omega \cos \lambda \cos \xi+\frac{\Omega^{2} r}{V} \cos \lambda(\cos \gamma \cos \lambda+\sin \gamma \sin \lambda \sin \xi) \\
\dot{\xi}=\frac{L \sin \sigma}{m V \cos \gamma}-\frac{V}{r} \cos \gamma \cos \xi \tan \lambda & \quad+2 \Omega(\tan \gamma \cos \lambda \sin \xi-\sin \lambda)-\frac{\Omega^{2} r}{V \cos \gamma} \sin \lambda \cos \lambda \cos \xi
\end{aligned}
$$

where $g=G M / r^{2}$ is the inverse-square gravitational acceleration, $r=R_{E}+h$ is the radial position measured from the center of the Earth, $\mu$ is the geocentric longitude, $\lambda$ is the geocentric latitude, $V$ is the total airspeed (i.e. velocity magnitude), $\gamma$ is the vehicle's flight path angle (FPA), and $\xi$ is the vehicle's heading angle. The lift and drag forces are represented as $\mathrm{L}$ and $\mathrm{D}$, respectively, given by

$$
\begin{aligned}
& L=\frac{1}{2} \rho(r) V^{2} C_{L}(\alpha, M) S_{r e f}=\frac{1}{2}\left(\rho_{0} e^{-\beta\left(r-r_{0}\right)}\right) V^{2} C_{L}(\alpha, M) S_{r e f} \\
& D=\frac{1}{2} \rho(r) V^{2} C_{D}(\alpha, M) S_{r e f}=\frac{1}{2}\left(\rho_{0} e^{-\beta\left(r-r_{0}\right)}\right) V^{2} C_{D}(\alpha, M) S_{r e f}
\end{aligned}
$$

where the term $\frac{1}{2} \rho(r) V^{2}$ is the dynamic pressure, $\bar{q}$, and the term $\rho_{0} e^{-\beta\left(r-r_{0}\right)}$ is a two-parameter model for atmospheric density, $\rho$, as a function of scaled altitude.

The aerodynamic coefficients of lift $\left(\mathrm{C}_{\mathrm{L}}\right)$ and drag $\left(\mathrm{C}_{\mathrm{D}}\right)$ are generated based on the $\mathrm{X}-33$ data. These coefficients are functions of Mach number and angle-of-attack (AoA) that are approximated by fitting the data with $2^{\text {nd }}$-order polynomials

$$
\begin{aligned}
& C_{L}(\alpha, M)=-0.0005225 \alpha^{2}+0.03506 \alpha-0.04857 M+0.1577 \\
& C_{D}(\alpha, M)=0.0001432 \alpha^{2}+0.00558 \alpha-0.01048 M+0.2204
\end{aligned}
$$


The additional parameters are defined as follows:

$$
\begin{aligned}
m & =2455 \mathrm{slugs} & & : \text { vehicle’s empty mass } \\
S_{\text {ref }} & =1608 \mathrm{ft}^{2} & & : \text { vehicle's reference planform area } \\
\rho_{0} & =0.002378 \mathrm{slugs} / \mathrm{ft}^{3} & & : \text { standard sea-level density } \\
r_{0} & =20902900 \mathrm{ft} & & : \text { atmospheric reference height } \\
\beta & =4.20168 \mathrm{e}-5 \mathrm{ft}^{-1} & & : \text { inverse atmospheric scale height } \\
G M & =0.14076539 \mathrm{e} 17 \mathrm{ft}^{3} / \mathrm{s}^{2} & & : \text { Earth's gravitational constant } \\
\Omega & =7.2722 \mathrm{e}-5 \mathrm{rad} / \mathrm{s} & & : \text { Earth's angular velocity } \\
g_{0} & =32.174 \mathrm{ft} / \mathrm{sec}^{2} & & : \text { standard gravity } \\
R_{e} & =20925646.3255 \mathrm{ft} & & : \text { Earth's radius }
\end{aligned}
$$

To fully capture the state at any instant, the variables in Eq. (1) form a six-dimensional state vector given as

$$
\mathbf{x}=[r, \mu, \lambda, V, \gamma, \xi]^{T} \in \mathbb{R}^{6}
$$

with the controls as the AoA $(\alpha)$ and the bank angle $(\sigma)$ such that

$$
\mathbf{u}=[\alpha, \sigma]^{T} \in \mathbb{R}^{2}
$$

Without a thrust force for the reentry gliding problem, the only controllable parameters for the 3-DOF model are the lift and drag forces. Typically, for symmetric flight (i.e. coordinated turns with zero sideslip angle, $\beta$ ), the lift and drag coefficients can be determined by the vehicle's AoA and Mach number, a function of velocity and speed-ofsound at a given altitude. However, it is the physical modulation of AoA and bank angle (BA) that controls the vehicle's translational motion through the atmosphere. Therefore, a common control vector for the reentry problem is that given by Eq. (7). Of course, this control vector assumes that there are no command delays (i.e. lags) and is sometimes referred to as "inertialess" control. To add more realism to the problem, as explained in Refs. 12 and 21, the rate limits are modeled by forming a new "virtual" control vector mathematically expressed as

$$
\mathbf{u}=\left[\begin{array}{ll}
u_{\alpha} & u_{\sigma}
\end{array}\right]^{T} \in \mathbb{R}^{2}
$$

Now, the original state vector must include the physical controls, $\alpha$ and $\sigma$, to form a new state vector:

$$
\mathbf{x}=\left[\begin{array}{lllllll}
r \mu & \lambda & \gamma & \xi & \alpha & \sigma
\end{array}\right]^{T} \in \mathbb{R}^{8}
$$

Remark: For a real vehicle, or in a 6-DOF simulation, it is the control surface deflections that create body moments and forces to augment the wind-relative AoA, BA, and sideslip angle.

To simulate external disturbances for this analysis, a wind gust model must be defined and integrated into the system.

\section{B. Wind Gust (Disturbance) Model}

The most common form of external disturbance encountered during atmospheric flight is the changing direction and intensity of the air mass itself (i.e. wind). There are various forms of wind and numerous ways to model wind. For this work, a constant-magnitude wind gradient approximation is adopted as a disturbance model. 


\section{Wind Gradient Approximation in Equations of Motion}

Typical aircraft and wind disturbance analysis employs an analytical approximation to the wind's flow field characteristics. For example, a windshear/downdraft can be modeled as

$$
\begin{aligned}
& W_{x}=w A(x) \\
& W_{h}=w \frac{h}{h_{*}} B(x)
\end{aligned}
$$

where $w$ is the intensity of the windshear/downdraft combination; the functions $A(x)$ and $B(x)$ represent the wind gradients as shown in Fig. 1; and $h^{*}$ is a reference altitude based on the range of validity. At lower altitudes, these wind velocity components typically form a wind shear that is commonly represented by a parabolic gradient as a function of altitude.

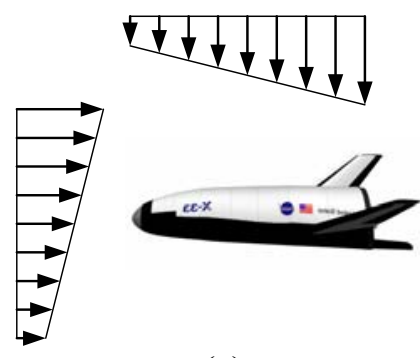

(a)

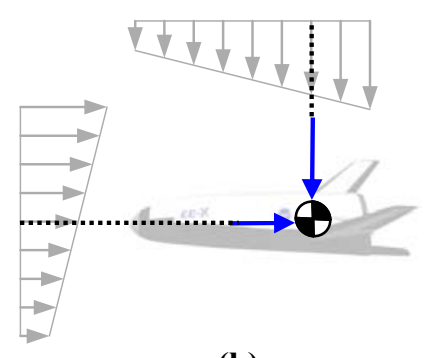

(b)

Fig. 1 Approximation of Horizontal and Vertical Wind Gradients Acting on Vehicle

Although various wind models (both discrete and continuous) and modeling standards exist, such as numerous wind gust models, a standard Dryden Wind Turbulence Model, and a standard Von Karman Wind Turbulence Model as specified by Military Specification MIL-F-8785C (Nov 1980) and Military Handbook MIL-HDBK-1797, this study uses a simplified approach to model a wind gust as illustrated in Fig. 1 (b).

Assuming a flat-earth approximation to the equations of motion, wind is often modeled by adding wind velocity components in the Cartesian x,y,z-directions $\left(W_{x}, W_{y}, W_{z}\right)$ to the kinematical equations such that

$$
\begin{aligned}
& \dot{x}=V \cos \gamma \cos \xi+W_{x} \\
& \dot{y}=V \cos \gamma \sin \xi+W_{y} \\
& \dot{z}=V \sin \gamma+W_{z}
\end{aligned}
$$

and likewise, the wind acceleration components $\left(\dot{W}_{x}, \dot{W}_{y}, \dot{W}_{z}\right)$ are added to the dynamical equations of motion

$$
\begin{gathered}
\dot{V}=-\frac{D\{V, z\}}{m}-g \sin \gamma-\left(\dot{W}_{x} \cos \gamma+\dot{W}_{z} \sin \gamma\right) \\
\dot{\gamma}=\frac{L\{V, z\} \cos \sigma}{m V}-\frac{g \cos \gamma}{V}+\frac{1}{V}\left(\dot{W}_{x} \sin \gamma-\dot{W}_{z} \cos \gamma\right) \\
\dot{\xi}=\frac{L\{V, z\} \sin \sigma}{m V \cos \gamma}
\end{gathered}
$$


that when combined, form a new set of equations for the closed-loop implementation. For this study, details of the wind gradient are not included in the model, but rather uniform, constant components are used in the dynamical equations such that the $\dot{W}$ terms are zero. Note that the actual implementation of the wind model equations involved the coordinate transformation from Cartesian to Spherical wind components such that $\left[W_{x}, W_{y}, W_{z}\right] \rightarrow\left[W_{\mu}, W_{\lambda}, W_{r}\right]$. Now, with the wind modeled directly into the vehicle's kinematical equations, only the direction and magnitude of the representative wind gust is required. Although arbitrarily selecting winds will suffice for this study, it is common that disturbance rejection analysis incorporates a worse case scenario in order to test the robustness of the proposed design.

\section{Worse-Case Winds}

To study the effects of wind on atmospheric flight vehicles, it is important to consider a worst-case scenario. A "nominal" wind depends on a particular region of the atmosphere, current temperature and pressure conditions, and geographical location. Unfortunately, for a vehicle transcending large vertical and horizontal distances through the atmosphere, many wind uncertainties exist. Although historical data can be used to chart trends, sudden and local variations in wind direction and intensity (e.g. turbulence and wind shear) make wind prediction nearly impossible. Because of these uncertainties and unpredictability, it is important to test for the worse possible case and in most design applications, to even add a margin of safety by exaggerating the worse-case.

Upper air winds, or winds aloft, over the continental United States typically flow from west to east as illustrated by the "westerly" velocity vectors in Fig. 2 showing long-term means (1968-1996) for May (a) and December (b). The troposphere, extending from the surface of the Earth to about 5-9 miles (26,400-47,520 ft) in elevation is the densest region of the atmosphere where most weather phenomena occurs. ${ }^{22}$ It is the high upper region of the troposphere where the jet stream velocity can reach approximately 80 to $180 \mathrm{mph}$ heading east.

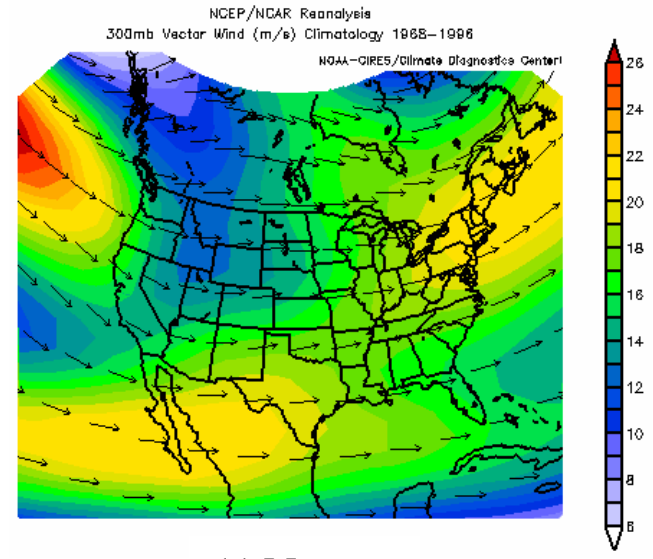

(a) May

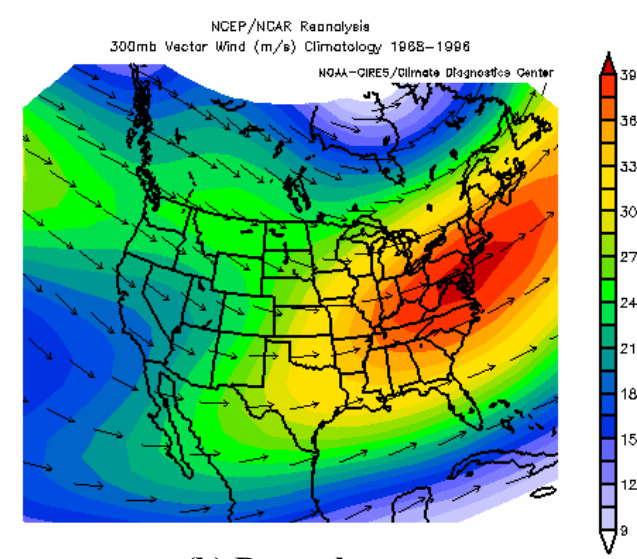

(b) December

Fig. 2 Example of Wind Flow Patterns over Continental US at 300mb $(\sim 30,000 \mathrm{ft})^{23}$

The wind components used in this demonstration represent a south-easterly flow which corresponds to a northwestward heading direction with the addition of a large vertical downward component. For winds aloft, a downward vertical component of wind is typically very small, on the order of $1-3 \mathrm{~cm} / \mathrm{s} .{ }^{\S}$ In localized storm systems this vertical component may exceed $25 \mathrm{~m} / \mathrm{s}$ ( $>82 \mathrm{ft} / \mathrm{s}$ ). ${ }^{* *}$ For the continental United States, this direction and magnitude of flow is commonly encountered in hurricane-force winds typically in the vicinity of the Gulf of Mexico. In this worse case scenario, a microburst or significant wind shear in the form of a column of sinking air is capable of generating wind speeds higher than $160 \mathrm{mph}^{24}$ Since vertical windshears mostly occur at altitudes below $1000 \mathrm{ft}$,

\footnotetext{
$\S$ David Brown, Private Communications, Department of Meteorology, Naval Postgraduate School, May 2006.

${ }^{* *}$ Robert Creasey, Private Communications, Department of Meteorology, Naval Postgraduate School, May 2006.
} 
they are more commonly encountered during takeoffs and landings; however, there are cases of large vertical winds at higher altitudes. A relevant Space Shuttle example is the uncommon but problematic occurrence of large vertical wind shears during interactions between the Polar Jet and the Sub-Tropical Jet near the base of the jet stream $(\sim$ 25,000 to $35,000 \mathrm{ft}$ ) over the south-eastern US.

\section{Wind Vector Selection}

Since large vertical wind shear is problematic near the Jet Stream, the wind vector for this study has a substantial vertical component. For the given initial conditions and final conditions corresponding to landing at the Space Shuttle Landing Facility (KTTS), Kennedy Space Center, FL, the wind is applied over a period of 20 sec beginning $460 \mathrm{sec}$ into the flight. This time-of-flight duration (460 to $480 \mathrm{sec}$ ) corresponds to an altitude of approximately 45,000 to 30,000 ft, overlapping the high upper troposphere where the Jet Stream reaches its peak. Also, this altitude corresponds to the region of transition (tropopause) from the upper atmosphere (stratosphere) to the lower atmosphere (troposphere) where large wind shear phenomena is likely to occur.

Based on averaged wind data from 1979 to 1998 climatology charts for the south-eastern US region, ${ }^{25}$ a nominal wind magnitude of about $30 \mathrm{kts}$ is appropriate, so for this study, a magnitude of $33.25 \mathrm{kts}$ was selected as the initial "light” wind, see Table 1.

For trajectory analysis, Table 1 shows the wind gusts selected for comparisons to a no wind scenario.

Table 1 Wind Gust Selection for Trajectory Comparisons

\begin{tabular}{lcccccc}
\hline \hline & \multicolumn{3}{c}{ Wind Components, fps } & \multicolumn{3}{c}{ Wind Magnitude } \\
\cline { 2 - 7 } \multicolumn{1}{c}{ Type } & $\mathrm{Wx}$ & $\mathrm{Wy}$ & $\mathrm{Wz}$ & $\mathrm{fps}$ & $\mathrm{kts}$ & $\mathrm{mph}$ \\
\hline Light & -30.00 & 15.00 & -45.00 & 56.12 & 33.25 & 38.27 \\
Moderate & -67.50 & 33.75 & -101.25 & 126.28 & 74.82 & 86.10 \\
Severe & -101.25 & 50.63 & -151.81 & 189.37 & 112.20 & 129.12 \\
Exaggerated & -151.88 & 75.94 & -227.81 & 284.13 & 168.34 & 193.73 \\
\hline \hline
\end{tabular}

Based on the Saffir-Simpson Hurricane Scale for wind speed categories, ${ }^{26}$ the worse-case scenario, labeled "exaggerated" in Table 1, corresponds to wind gust intensity in the "Cat 5" hurricane range (> $155 \mathrm{mph})$. Note that although it is labeled "exaggerated" in this study, it is not unrealistic. As discussed later, results show that the vehicle’s flight guidance algorithm can tolerate this significant wind gust intensity.

\section{Determination of Maximum Wind (Disturbance Rejection Performance)}

With testing any control algorithm, it is important to analyze the controller's disturbance rejection performance and estimate what maximum disturbance the feedback system can tolerate until the system in no longer controllable. For this study, the system's performance is numerically tested. In order to determine how much wind the vehicle can handle before the optimal feedback guidance algorithm fails to converge, various wind profiles are implemented with increasing magnitude. For purpose of comparison, the wind direction is fixed by using the same direction cosine (i.e. same unit vector) and scaling the velocity magnitude, at most, by $50 \%$ increments. Increasing the winds until problems occur (e.g. constraint violations or non-convergence) helps indicate the maximum allowable windgust magnitude.

\section{Problem Formulation}

\section{B. Reentry Optimal Control Problem Formulation}

Even from a sub-orbital entry altitude, the reentry vehicle (RV) must traverse a fairly large portion of the Earth's surface, descend through the Earth's atmosphere, and reach a designated landing site with the appropriate velocity and attitude. In principle, it is possible to use the entry guidance algorithm developed in this paper for autolanding as well; however, since our present model does not take into account aerodynamic ground effects, we focus our efforts in guiding the RV all the way up to a handover to autoland guidance. As a result of this, we discard the traditional 
notions of segmented guidance schemes that involve such concepts as terminal area energy management (TAEM) schemes; instead, we define a Final Approach Corridor (FAC) as illustrated in Fig. 3. In this concept, it is required to get the vehicle aligned with the runway centerline at the appropriate altitude, velocity, etc. Thus, the FAC manifests a projection of all the final conditions onto one two-dimensional box. Therefore, the problem is then to find the control history that minimizes some cost function associated with intercepting the desired FAC. After all, the primary objective of RLV reentry is to safely land at a designated landing site. Heat, dynamic pressure, structural loads, drag, or some other criterion could be minimized, and these can be modeled as path constraints. The reentry problem is then formulated by appropriately defining the cost function and corresponding constraints.

A general expression for a terminal guidance performance index is defined by the following cost function,

$$
J\left(\underline{x}(t), \underline{u}(t), t_{0}, t_{f}\right)=\sum_{i=1}^{n} w_{i} \Delta s_{i}{ }^{2}=w_{1} \Delta s_{1}{ }^{2}+w_{2} \Delta s_{2}{ }^{2}+\ldots w_{n} \Delta s_{n}{ }^{2}
$$

where $\Delta s_{i}=\left|s_{2}-s_{1}\right|_{i}$ is the difference between a specific final state value, $x_{i}\left(t_{f}\right)$, and a desired final condition (i.e. the "target") for that state, and $w_{i}$ is the corresponding weighting term that can be adjusted depending on the relative importance of the condition.

For this example of using the FAC-target, the cost function can simply be the difference between the center of the FAC and the vehicle's final position in coordinates of altitude, latitude, and longitude designated as $\Delta r, \Delta \lambda, \Delta \mu$, respectively and equally weighted $\left(w_{1,2,3}=1\right)$ such that

$$
J(\cdot)=1 \cdot \Delta r^{2}+1 \cdot \Delta \lambda^{2}+1 \cdot \Delta \mu^{2}=\left|F A C_{r}-r_{f}\right|^{2}+\left|F A C_{\lambda}-\lambda_{f}\right|^{2}+\left|F A C_{\mu}-\mu_{f}\right|^{2}
$$

Note that the this expression can also include difference terms for the remaining states such as $V, \gamma$, and $\xi$.

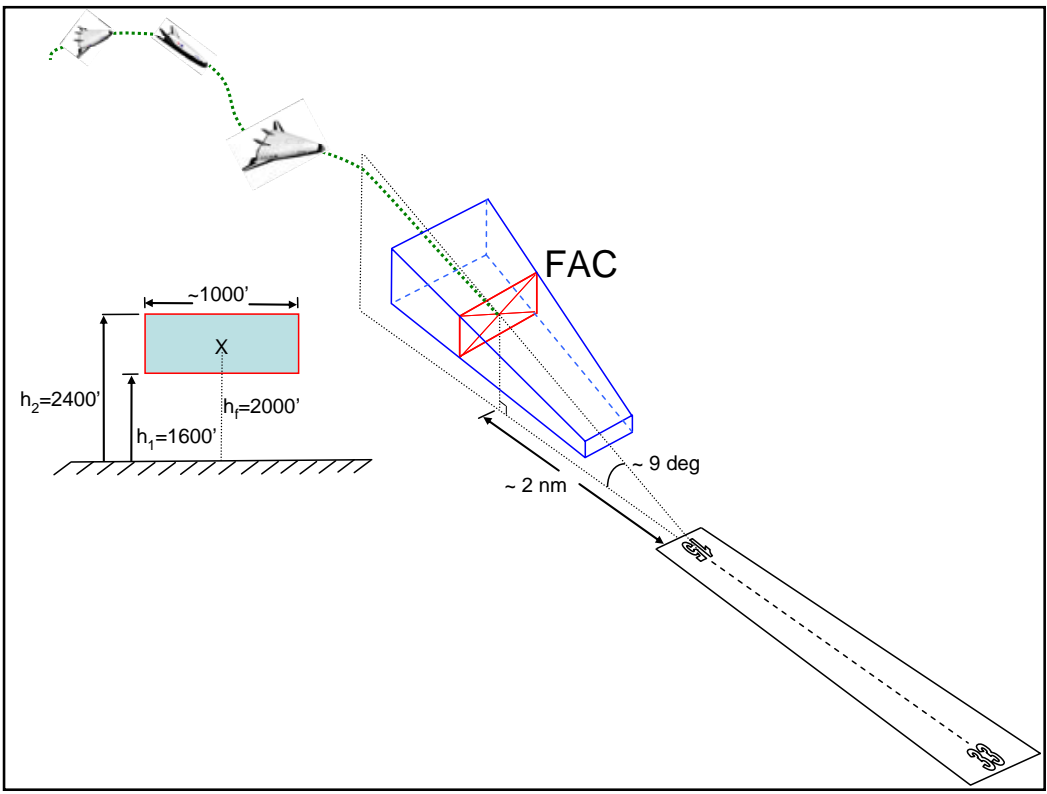

Fig. 3 Final Approach Corridor (FAC) for "Target" Conditions 
Thus, the specific optimal control formulation for this RV problem is stated as follows: Given an initial position vector $\left(\left[r_{0}, \mu_{0}, \lambda_{0}\right]\right)$, velocity magnitude $\left(V_{0}\right)$, FPA $\left(\gamma_{0}\right)$, heading angle $\left(\xi_{0}\right)$, AoA $\left(\alpha_{0}\right)$, and BA $\left(\sigma_{0}\right)$, find the control history $(\alpha, \sigma)$ that minimizes the miss distance to a predefined target under various constraints. Thus, the problem is to minimize $J(\cdot)$ subject to the dynamic constraints, Eq. (1), the initial and final event conditions specified as:

$$
\begin{aligned}
\left(t_{0}, h_{0}, \mu_{0}, \lambda_{0}, V_{0}, \gamma_{0}, \xi_{0}\right) & =\left(0 \mathrm{sec}, 167323 \mathrm{ft},-85 \mathrm{deg}, 30 \mathrm{deg}, 8530.2 \frac{\mathrm{ft}}{\mathrm{s}},-1.3 \mathrm{deg}, 0 \mathrm{deg}\right) \\
\left(h_{f}, \mu_{f}, \lambda_{f}, V_{f}, \gamma_{f}, \xi_{f}\right) & =\left(2000 \pm 400 \mathrm{ft},-80.7112 \pm 0.001371 \mathrm{deg}, 30 \pm 0.001097,300 \frac{\mathrm{ft}}{\mathrm{s}},-6.0 \mathrm{deg},-60 \mathrm{deg}\right) \\
- & 33.33 \frac{\mathrm{ft}}{\mathrm{s}} \leq \dot{h}_{f} \leq 8.33 \frac{\mathrm{ft}}{\mathrm{s}}
\end{aligned}
$$

and the state (17), path (18), and control (19) inequality constraints, respectively, specified as

$$
\begin{aligned}
& {\left[\begin{array}{c}
0 \mathrm{ft} \\
-90 \mathrm{deg} \\
-89 \mathrm{deg} \\
1.0 \mathrm{ft} / \mathrm{s} \\
-89 \mathrm{deg} \\
-180 \mathrm{deg}
\end{array}\right] \leq\left[\begin{array}{c}
h \\
\mu \\
\lambda \\
V \\
\gamma \\
\xi
\end{array}\right] \leq\left[\begin{array}{c}
400000 \mathrm{ft} \\
90 \mathrm{deg} \\
89 \mathrm{deg} \\
17060.4 \mathrm{ft} / \mathrm{s} \\
89 \mathrm{deg} \\
180 \mathrm{deg}
\end{array}\right]} \\
& {\left[\begin{array}{c}
-2.5 \mathrm{~g} ' \mathrm{~s} \\
0 \\
0
\end{array}\right] \leq\left[\begin{array}{c}
n_{z} \\
\bar{q} \\
Q
\end{array}\right] \leq\left[\begin{array}{c}
2.5 \mathrm{~g}^{\mathrm{s}} \\
300 \mathrm{lb} / \mathrm{ft}^{2} \\
70 \mathrm{BTU} / \mathrm{ft}-\mathrm{s}
\end{array}\right]} \\
& -10 \mathrm{deg} \leq \alpha \leq 50 \mathrm{deg} \\
& -80 \mathrm{deg} \leq \sigma \leq 80 \mathrm{deg}
\end{aligned}
$$

where the path constraint terms represent the normal acceleration $n_{z}=|L \cos \alpha+D \sin \alpha|$, the dynamic pressure $\bar{q}=\frac{1}{2} \rho(r) V^{2}$, and the heating rate $Q=k \sqrt{\rho(r)} V^{3.15}$ with $k=4.47228 e-9$ as the material heating coefficient (scaled).

The overall goal is to minimize the miss distance to the center of the FAC-target box, by defining the end-point conditions as the projected FAC-box constraints in terms of position, velocity, and flight path attitude. Also, to ensure the vehicle's sink rate is appropriate for final approach setup, a constraint on final vertical speed, $\dot{r}_{f}$, was imposed. Note that although this particular formulation does not use Eqs. $(8,9)$ nor place constraints directly on the control rates, the effects of doing so are shown in the results section. 


\section{Solving the Optimal Control Problem in Real Time}

\section{A. Necessary Conditions for Optimality}

To demonstrate optimality, a theoretical analysis is first performed based on optimal control theory. To demonstrate the necessary conditions needed for optimality the first step requires the formulation of the Hamiltonian:

$$
H(P, x, u, t)=F(x, u, t)+P^{T} f(x, u, t)
$$

where $F(\cdot)$ is the Lagrange cost, $\mathrm{P}$ represents a vector of Lagrange multipliers given by Eq. (21), and $f(\cdot)$ is the vector field for the right hand side of the differential equations of motion given in Eq. (1). Therefore, with

$$
\mathbf{P}=\left[P_{r}, P_{\mu}, P_{\lambda}, P_{V}, P_{\gamma}, P_{\xi}\right]^{T} \in \mathbb{R}^{6} ; \quad \mathbf{x}_{f}=\left[r_{f}, \mu_{f}, \lambda_{f}, V_{f}, \gamma_{f}, \xi_{f}, \dot{r}_{f}\right] \in \mathbb{R}^{7}
$$

and omitting the $\Omega$ - terms for brevity, the Hamiltonian becomes

$$
\begin{aligned}
& H(\cdot)=P_{r}[V \sin \gamma]+P_{\mu}\left[\frac{V \cos \gamma \cos \xi}{r \cos \lambda}\right]+P_{\lambda}\left[\frac{V \cos \gamma \sin \xi}{r}\right] \ldots \\
&+P_{V}\left[-\frac{1}{2 m} \rho_{0} e^{-\beta\left(r-r_{0}\right)} V^{2} C_{D}(\alpha, M) S_{r e f}-g \sin \gamma+\ldots\right] \ldots \\
&+P_{\gamma}\left[\frac{1}{2 m} \rho_{0} e^{-\beta\left(r-r_{0}\right)} V C_{L}(\alpha, M) S_{r e f} \cos \sigma+\left(\frac{V}{r}-\frac{g}{V}\right) \cos \gamma+\ldots\right] \ldots \\
&+P_{\xi}\left[\frac{1}{2 m} \frac{\rho_{0} e^{-\beta\left(r-r_{0}\right)} V C_{L}(\alpha, M) S_{r e f} \sin \sigma}{\cos \gamma}-\frac{V}{r} \cos \gamma \cos \xi \tan \lambda+\ldots\right] \\
& \dot{\mathbf{x}}=\frac{d H}{d \mathbf{P}} \Rightarrow R H S \text { of dynamic equations }
\end{aligned}
$$

The Hamiltonian Minimization Condition (HMC) is based on Pontryagin's Minimum Principle such that the optimal control must minimize the Hamiltonian with respect to control. For this problem, the control is subject to an inequality constraint so the Karush-Kuhn-Tucker (KKT) Theorem is applied by taking the Lagrangian of the Hamiltonian:

$$
\mathbf{H}(\ldots, u, q)=H(\cdot)+q^{T} h
$$

where $q$ is a KKT multiplier and $h$ is the control constraint vector, $\mathbf{u}:=[\alpha, \sigma]$. The appropriate necessary condition is:

$$
\frac{\partial \mathbf{H}}{\partial \mathbf{u}}=\frac{\partial H}{\partial \mathbf{u}}+\left(\frac{\partial h}{\partial \mathbf{u}}\right)^{T} q=0
$$

and substituting Eqs. (22), (4), (5) and the controls into (25), the HMC becomes 


$$
\left\{\begin{array}{l}
P_{V}\left[-\rho_{0} e^{-\beta\left(r-r_{0}\right)} V^{2}(0.0002 \alpha+0.006) S_{r e f} / 2 m\right]+\ldots \\
P_{\gamma}\left[\rho_{0} e^{-\beta\left(r-r_{0}\right)} V(-0.0010 \alpha+0.035) S_{r e f} \cos \sigma / 2 m\right]+\ldots \\
P_{\xi}\left[\rho_{0} e^{-\beta\left(r-r_{0}\right)} V(-0.0010 \alpha+0.035) S_{r e f} \sin \sigma / 2 m \cos \gamma\right]+q_{\alpha}=0 \\
P_{\gamma}\left[-\rho_{0} e^{-\beta\left(r-r_{0}\right)} V\left(-0.0005 \alpha^{2}+0.035 \alpha-0.049 M+0.16\right) S_{r e f} \sin \sigma / 2 m\right]+\ldots \\
P_{\xi}\left[\rho_{0} e^{-\beta\left(r-r_{0}\right)} V\left(-0.0005 \alpha^{2}+0.035 \alpha-0.049 M+0.16\right) S_{r e f} \cos \sigma / 2 m \cos \gamma\right]+q_{\sigma}=0
\end{array}\right.
$$

Also, the multiplier-constraint pair must satisfy the following KKT complementarity conditions (CC):

$$
q_{\alpha, \sigma}\left\{\begin{array}{ccc}
\leq 0 & \text { if } & u_{\alpha, \sigma}=-10,-89 \\
=0 & \text { if } & (-10,-89)<u_{\alpha, \sigma}<(50,89) \\
\geq 0 & \text { if } & u_{\alpha, \sigma}=50,89
\end{array}\right.
$$

To determine the final value of the Hamiltonian, the Endpoint Lagrangian, given as,

$$
\begin{aligned}
\mathbf{E}\left(v, x_{f}, t_{f}\right):= & E\left(x_{f}, t_{f}\right)+v^{T} e\left(x_{f}, t_{f}\right) \\
\Rightarrow & \left|F A C_{r}-r_{f}\right|^{2}+\left|F A C_{\lambda}-\lambda_{f}\right|^{2}+\left|F A C_{\mu}-\mu_{f}\right|^{2} \ldots \\
& +v_{r}\left(r_{f}-r^{f}\right)+v_{\lambda}\left(\lambda_{f}-\lambda^{f}\right)+v_{\mu}\left(\mu_{f}-\mu^{f}\right) \ldots \\
& +v_{V}\left(V_{f}-V^{f}\right)+v_{\gamma}\left(\gamma_{f}-\gamma^{f}\right)+v_{\xi}\left(\xi_{f}-\xi^{f}\right)
\end{aligned}
$$

is substituted into the Hamiltonian Value Condition (HVC):

$$
H\left(t_{f}\right)+\frac{\partial \mathbf{E}}{\partial t_{f}}=0 \Rightarrow H\left(t_{f}\right)=0
$$

This indicates that the final value for the Hamiltonian should be zero for this problem. Also, sometimes applying the Terminal Transversality Conditions (TTC)

$$
\lambda\left(t_{f}\right)=\frac{\partial \mathbf{E}}{\partial \mathbf{x}_{f}}
$$

may give some indication of the final value of the dual variables that can be used later to confirm numerical results. Likewise, the Hamiltonian Evolution Equation (HEE) is used to indicate the nature of the Hamiltonian with respect to time such that: 


$$
\frac{\partial H}{\partial t}=0 \Rightarrow \frac{d H}{d t}=0
$$

Here, the Hamiltonian is constant with respect to time. Combining HEE (31) with HVC (29), the Hamiltonian should be 0 for all time. The above analysis is used throughout this study to verify that the numerical results satisfy the necessary conditions for optimality as briefly discussed in the Results section to follow.

\section{B. Optimal Feedback Implementation}

Feedback is achieved through real-time computation of the optimal control problem. A schematic of this concept is illustrated in Fig. 4.

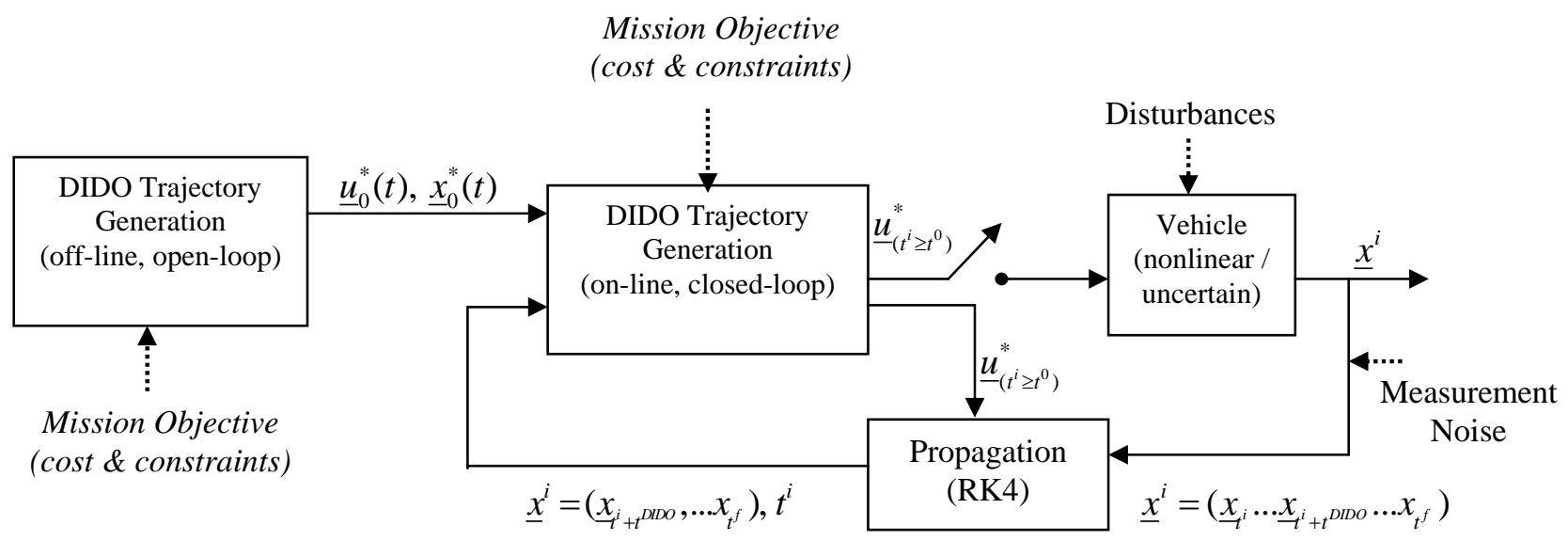

Fig. 4 Off-Line and On-Line Trajectory Generation Control Architecture

Figure 4 illustrates the simulation technique where a Runge-Kutta propagation scheme is used in conjunction with measurement noise and external disturbances to integrate the equations of motion and determine the current vehicle state vector. Thus, the RK propagation for the disturbed and uncertain vehicle simulates a plant. Complete details on such a method for guidance and control are described in Ref. 16.

\section{Numerical Results}

\section{A. Open-Loop Solution}

For the reentry trajectory, the open-loop terminal guidance solution, shown in Fig. 5, takes only about 30 seconds to generate from an arbitrary starting point. Although 30 seconds may be viewed as rapid in the context of "setup time," this computation time can be reduced by at least a factor of 100 by optimizing the actual code, eliminating the Windows and MATLAB overhead etc. ${ }^{27}$ None of these computational enhancements were carried out because the purpose of the current work is to demonstrate the principles. In any case, Fig. 5 shows that the optimal open-loop control given by the $\alpha, \sigma$-rate modulation drives the RV to the FAC-target box over a total flight time of 669.1 seconds and within the allowable tolerance for the desired end-point conditions. 

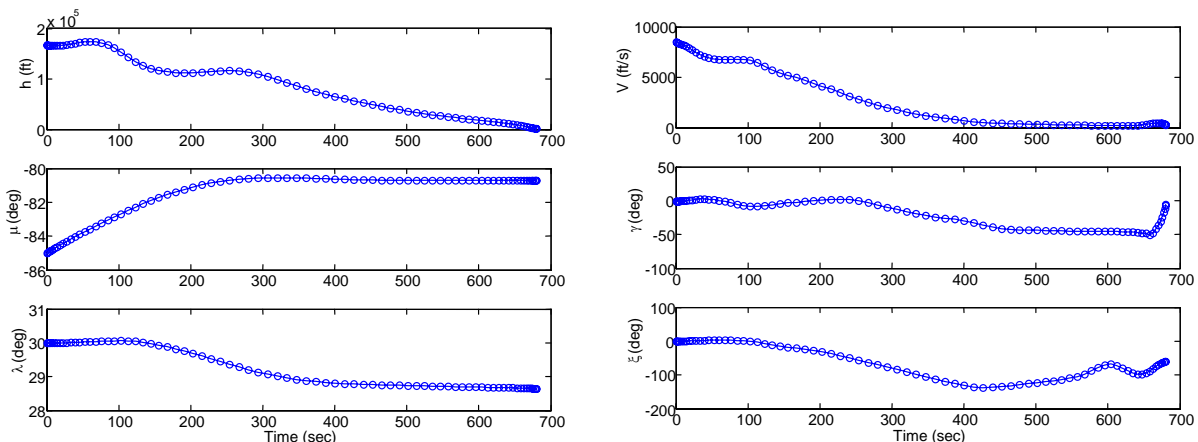

(a) States (position)

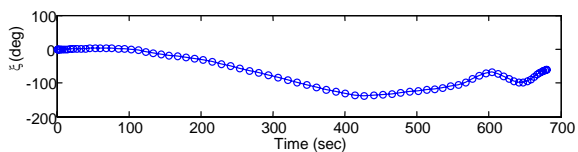

(b) States (velocity)
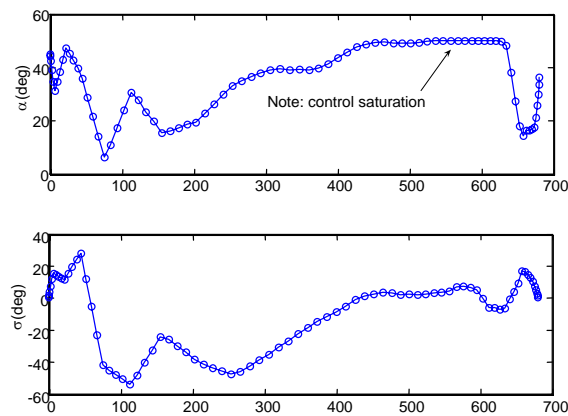

(c) Controls

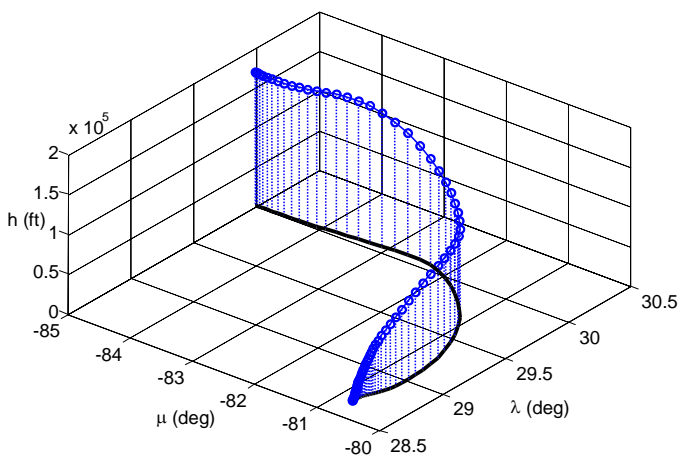

(d) 3-D Reference Trajectory

Fig. 5 Optimal Open-Loop Solution (No Wind)

\section{B. Feasibility}

The feasibility of the computational solution can be independently validated by comparing the DIDO results to the propagated states via a separate ODE Runge-Kutta propagator. By interpolating the values of the control function, $\mathrm{u}\left(\mathrm{t}_{\mathrm{i}}\right)$, at the LGL points and then integrating the differential dynamical equations, $\dot{x}=f(x, u(t), t)$, via MATLAB's ode45 solver, a comparison of error norms can be made with the DIDO trajectory results. Although not included here, results showed that the open-loop system response does satisfy the end-point conditions within an acceptable error range.

\section{Optimality}

One way to demonstrate optimality is by verifying that the necessary optimality conditions are satisfied. This is performed by analytically solving some of the necessary conditions and then comparing the analytic results with the numerical solutions. In the absence of useful costate information, Bellman's Principal of Optimality can be applied to validate optimality. Both of these methods are presented in the proceeding sections.

\section{Optimality via Necessary Conditions}

Comparing the numerical results to the above theoretical analysis of the HVC, HEE, HMC, and TTC conditions validates the optimality of the computational solution. The HVC stated in the theoretical analysis indicates that the Hamiltonian should be 0 at the final time (i.e. $\mathrm{H}\left(\mathrm{t}_{\mathrm{f}}\right)=0$ ). From the HEE, it can be shown that the Hamiltonian is constant with respect to time. Combining these two conditions, the Hamiltonian should be 0 for all time, clearly evident in Fig. 6. Likewise, the Lagrange multipliers for the dynamics at the final time match the theoretical TTC results (not shown). 


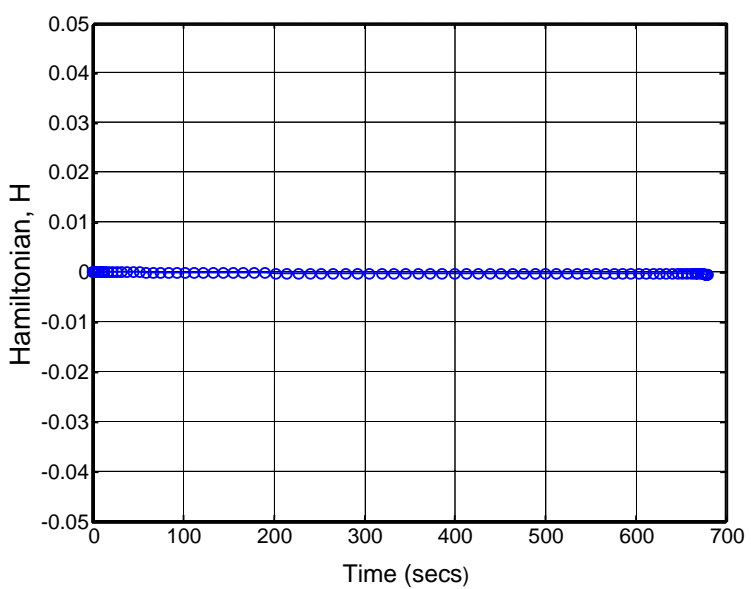

Fig. 6 Open-loop Hamiltonian

\section{Optimality via Bellman's Principle}

Another test to confirm computational optimality is to apply Bellman's Principle of Optimality. This principle essentially states that by using any point on the original optimal trajectory as an initial condition to a new problem, with all other problem formulation parameters the same, should result in the same optimal trajectory with the same or better cost. Although it is not shown here, this method was used to validate the open-loop optimality and is demonstrated in the following closed-loop implementation.

\section{Closed-Loop Solution}

Although the previous open-loop solution results in a feasible trajectory and desired end-point conditions that satisfy the necessary conditions for optimality, it does not account for any external disturbances as would be the case in real applications. To illustrate the effects of external disturbances for this reentry problem, a simulated wind gust was applied over a period of 20 seconds beginning at 460 seconds into the flight. Sensor measurement errors and parameter uncertainty can be simulated by assuming the role of the errors from the numerical propagation. The effects of the wind on the open-loop (OL) trajectory are seen in Figs. 7 and 8.
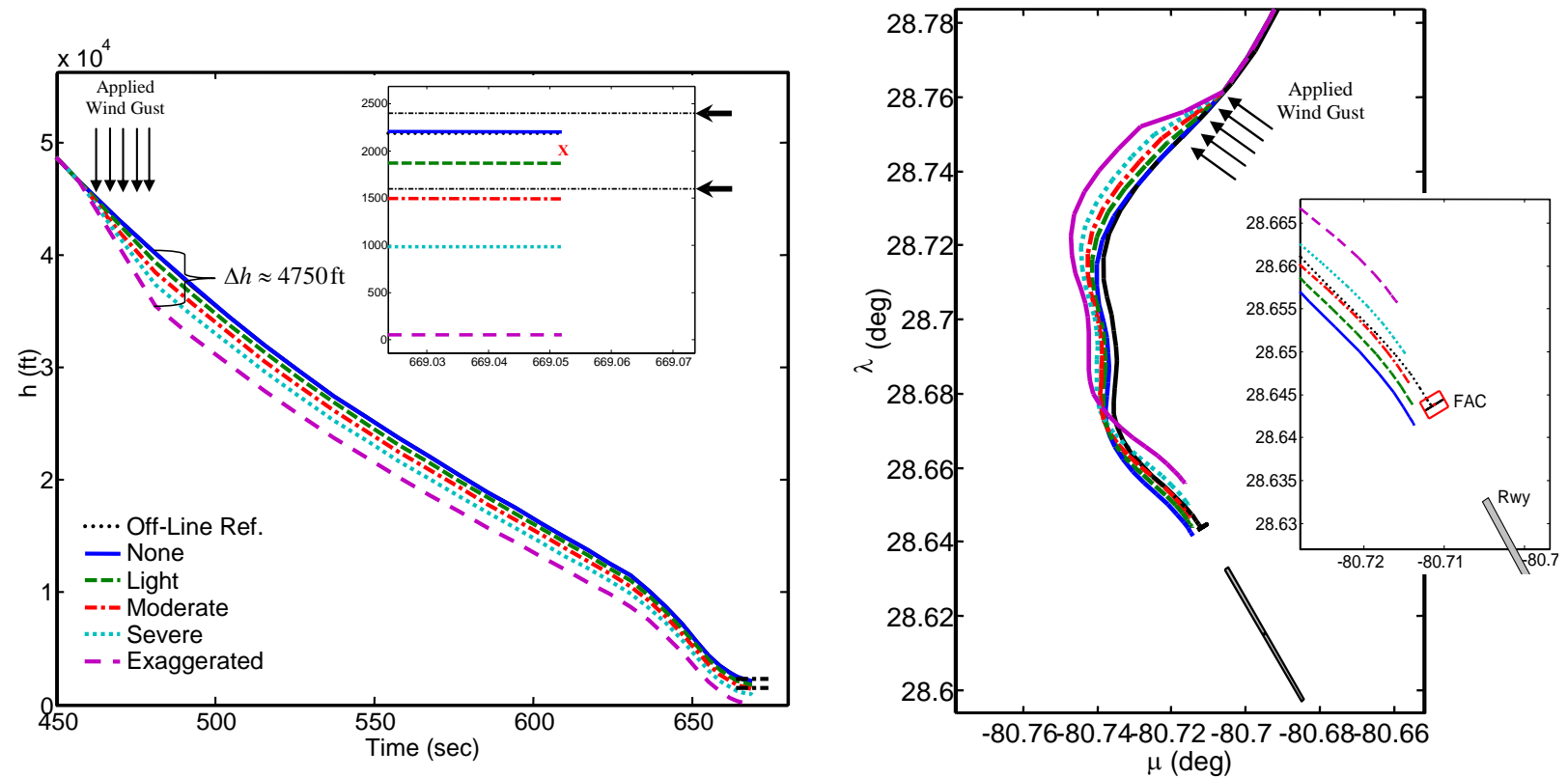

Fig. 7 Effect of Wind Gusts on Position for Open-Loop (OL) System 


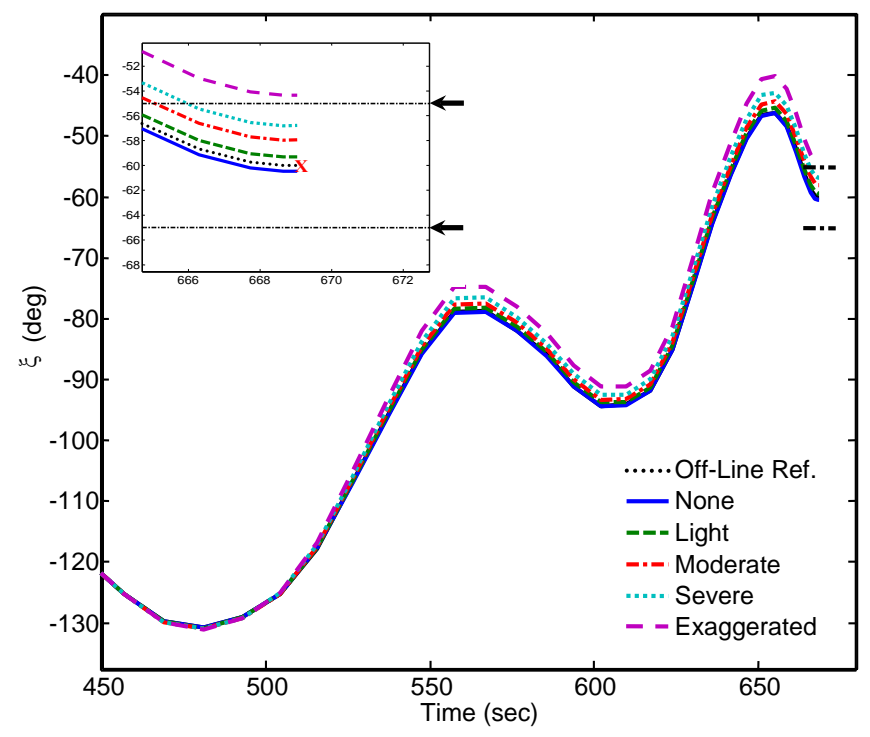

Fig. 8 Effect of Wind Gusts on Heading for Open-Loop (OL) System

Clearly shown by the insets, increasing the wind-gust intensity pushes the vehicle further from the intended target as indicated by the "x"-mark. Figure 7 shows that an "exaggerated" wind-gust (i.e. > Cat 5 hurricane) causes a rapid decrease in altitude of almost $5000 \mathrm{ft}$. Figure 8 shows that even with wind gusts up to and including "severe," the final heading is still within limits as indicated by arrow-marks on the inset. Some errors and disturbances are acceptable because the FAC-target design allows margin on the vehicle's final conditions; however, with large disturbances such as the "exaggerated" wind gust, the system is not capable of compensating without feedback. The 2-D ground track in Fig. 7 is a good example of how other errors in the system (e.g. modeling, numerical propagation, etc) can negatively affect the trajectory. Here, even the no-wind case fails to hit the FAC-target box. It is evident that feedback is required to handle these uncertainties and disturbances. Fig. 9 shows the effects of wind gusts on the closed-loop (CL) trajectory for the variables of interest.
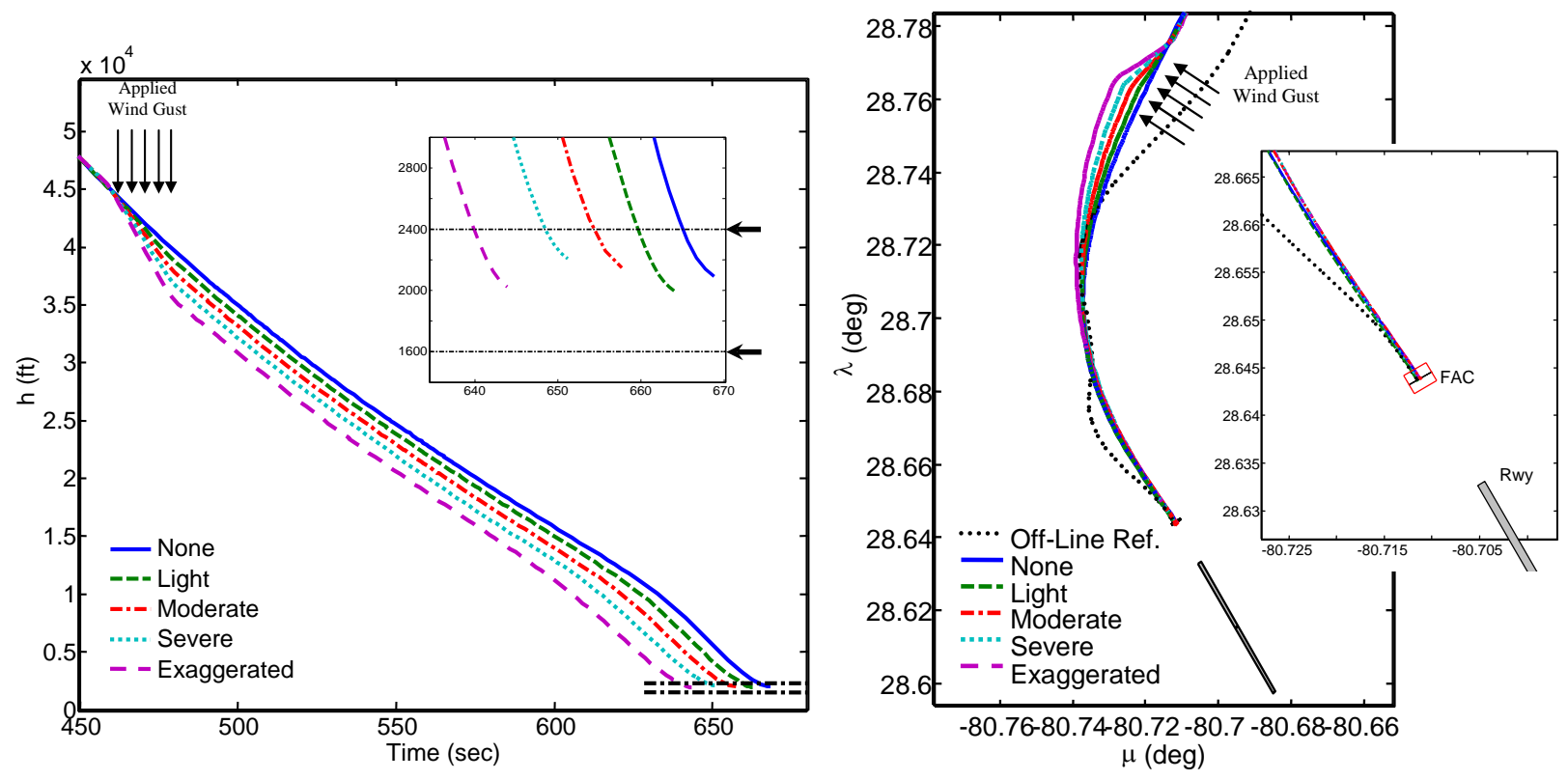

Fig. 9 Effect of Wind Gusts on Position for Closed-Loop (CL) System

American Institute for Aeronautics and Astronautics 
In contrast to the OL results, it is clear that all the closed-loop trajectories satisfy the final conditions, including the "exaggerated" case with a wind-gust magnitude of $194 \mathrm{mph}$ ! Also note how the 2-D ground track nicely converges to the center of the FAC-target in Fig. 9. Note that the vehicle is not guided along a reference trajectory. In fact, we do not use the concept of reference trajectory tracking; rather, the principle we adopt is that of autonomous real-time trajectory generation.

With larger disturbances there are signs of control "chatter" as indicated in Fig. 10; however, note that this is not chattering but rather an artifice of compressed x-axis scaling. In this case, none of the "spikes" exceed a realistic rate of $5 \mathrm{deg} / \mathrm{s}$.
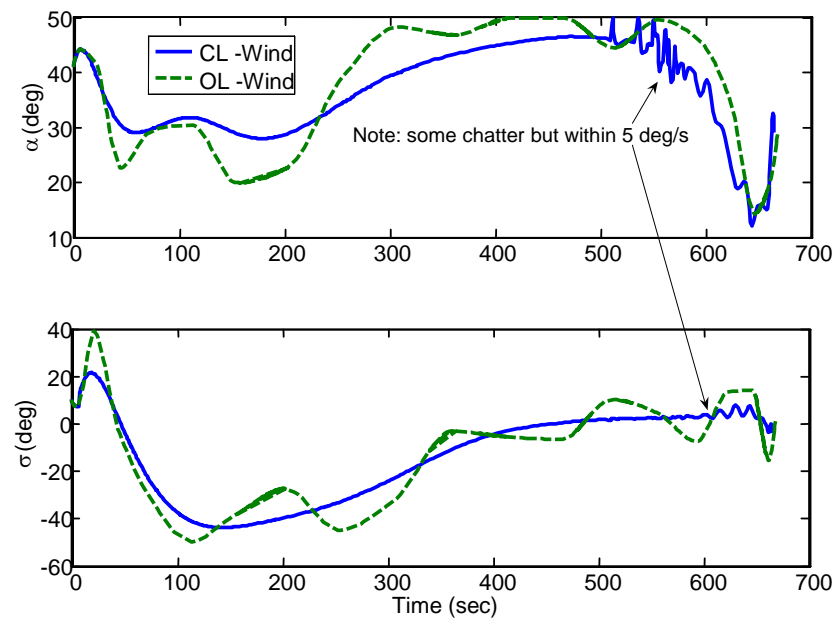

Fig. 10 Example of Control "Chatter" for Closed-Loop System

In order to demonstrate the principles described in the Modeling section (see Eq. 8), we investigate the effect of limiting the control rates by imposing rate constraints. Figure 11 shows an example of a similar scenario as in Fig. 10, but without experiencing any control "chatter."
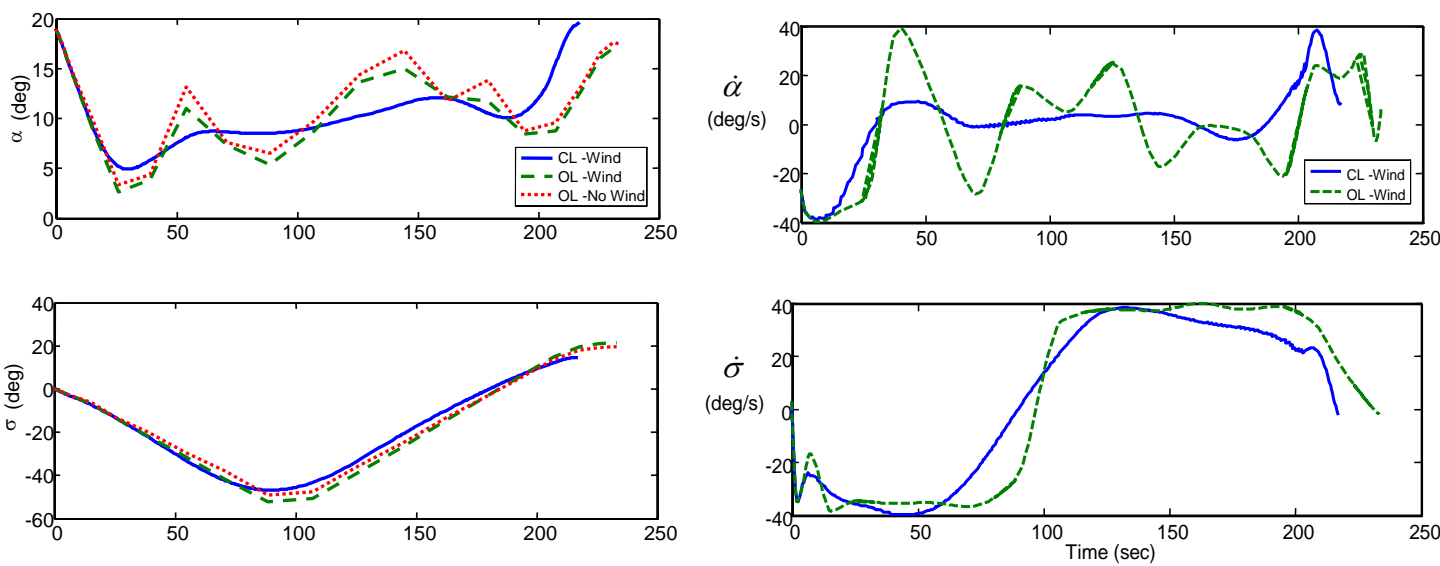

Fig. 11 Example of Modeling Rates as "Virtual" Controls to Eliminate "Chatter"

Results from a similar model, but without path constraints, reveals that a wind gust of approximately $206 \mathrm{ft} / \mathrm{sec}$ ( $140 \mathrm{mph}$ ) will cause the vehicle to impact the earth's surface unless the closed-loop algorithm is implemented. Shown in Fig. 12, the vehicle flying the open-loop trajectory is slammed into the ground, whereas the closed-loop 
trajectory corrects for the microburst and is able to achieve a final altitude of $1629 \mathrm{ft}$, within the FAC-box vertical limits of $2000 \pm 400 \mathrm{ft}$.

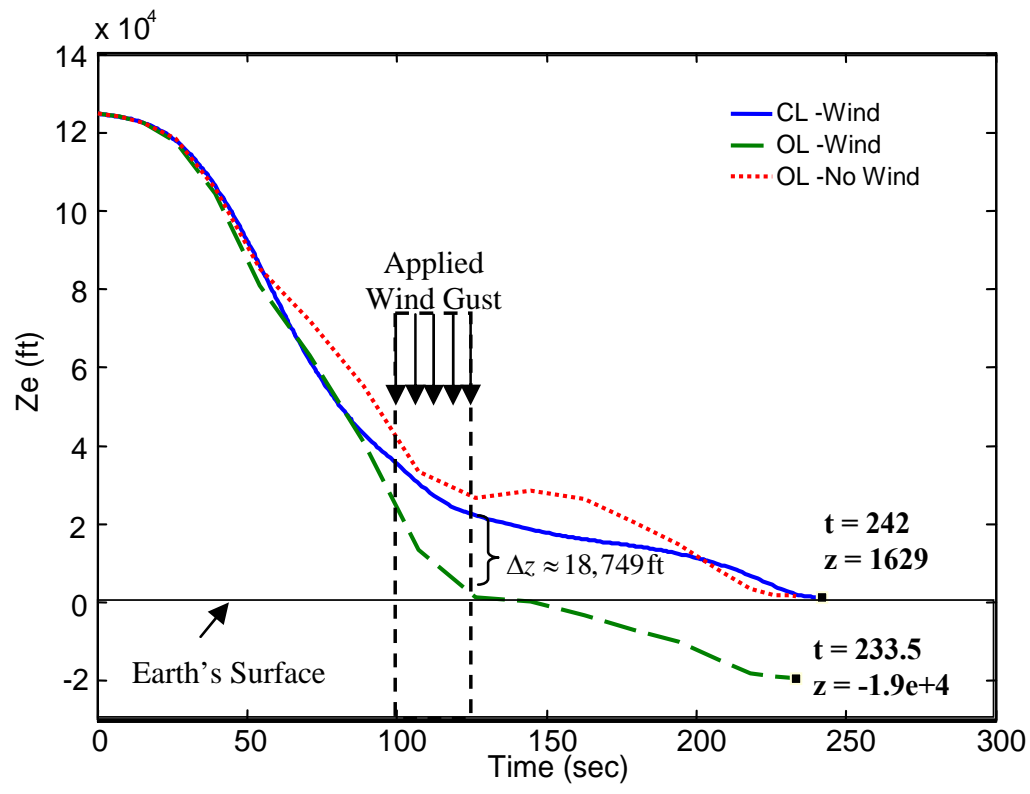

Fig. 12 Worse-Case Scenario: “Exaggerated” Wind Gust Causing Earth Impact

Both cases (Figs. 9 \& 12) demonstrate that a viable trajectory exists under hurricane-force wind gusts when implementing the optimal nonlinear feedback. Also, like the case for no disturbances, it is important to note that for small disturbances, such as the light-wind scenario, the closed-loop trajectory approximately tracks the off-line open-loop reference trajectory. This is a direct result of Bellman's Principle. Since the initial condition to the reoptimization problem lies approximately on the original optimal path, then the new solution will be along the same trajectory. This "stability" result is a direct consequence of optimality. ${ }^{28}$

As with all reentry problems, it is important to ensure that the vehicle does not exceed limits on dynamic pressure, heating, and structural loads. A benefit of our constrained optimal feedback guidance is that the path constraints are not violated as demonstrated in Figs. 13 and 14.

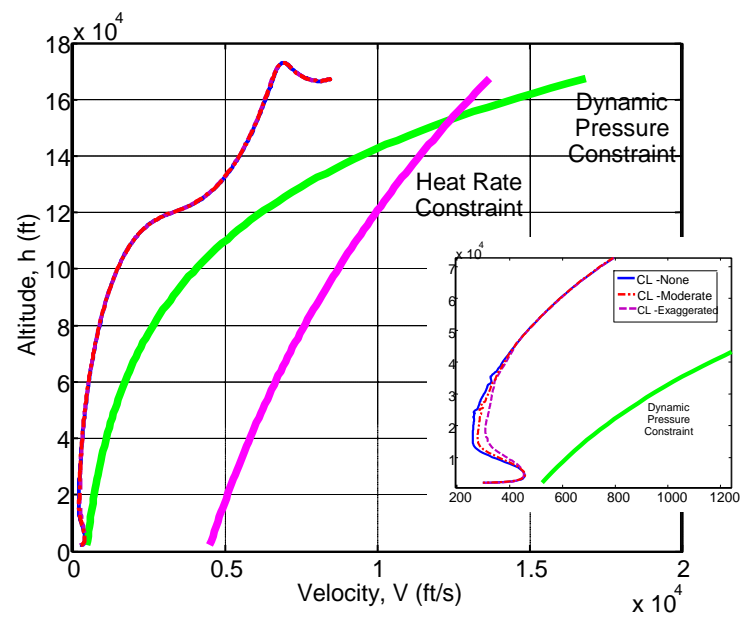

Fig. 13 Velocity vs. Altitude and Path Constraints with Wind Disturbance
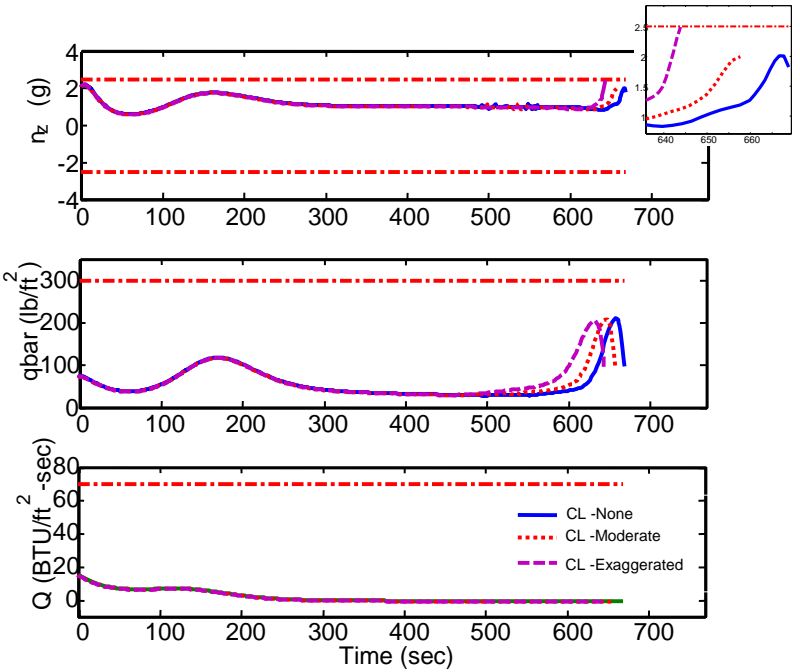

Fig. 14 Comparison of Path Function Time Histories

17

American Institute for Aeronautics and Astronautics 
Depending on the specific initial conditions, the resulting flight path may or may not have active path constraints. Figure 13 shows how a sample of CL trajectories do not cross the plotted dynamic pressure and heat rate constraints and also have a margin of safety. Note that this margin of safety can also be explicitly enforced.

\section{E. Computational Time}

Success of this feedback method depends heavily on relatively fast computation time. A theorem that links the required computation time to the dynamics of the system is developed in Ref. 16. Computation of the first off-line, open-loop optimal trajectory takes an average of 12 seconds and the subsequent open-loop optimal control updates are computed within approximately 1.2 to 9.75 seconds depending on the disturbance induced by the wind gusts, and the stability of the Windows environment at the time of run. These trajectories were generated on a Dell Optiplex Desktop computer with a Pentium M, 3.40 Ghz processor, and 1.0 GB of RAM. The feedback computation times are shown over the entire trajectory in Fig.15(a)-(e).

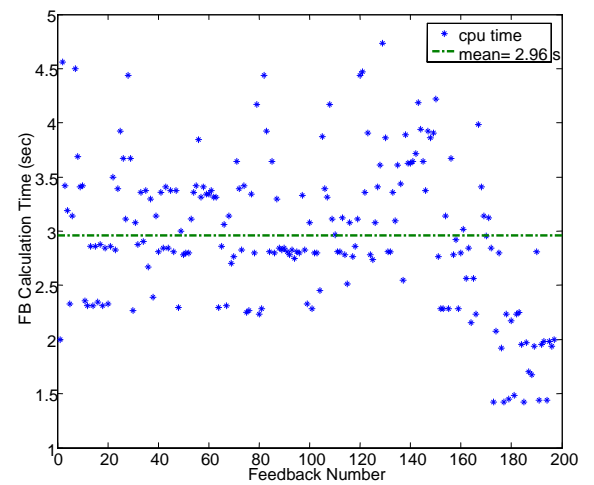

(a) No Wind

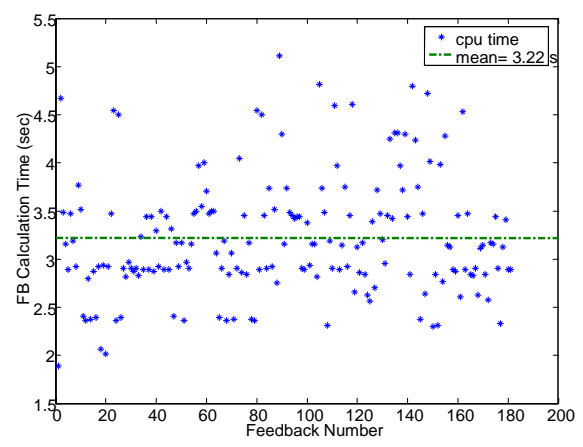

(b) Light Wind

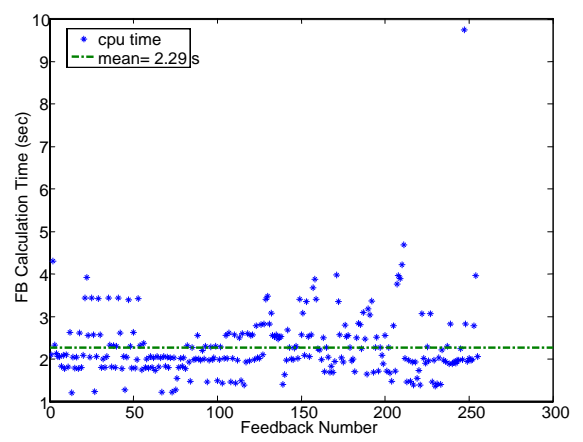

(d) Severe Wind

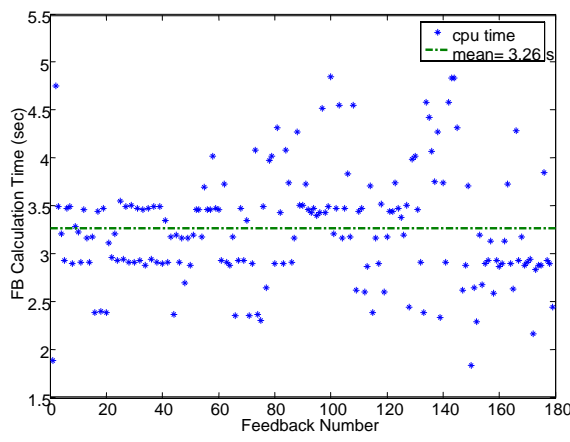

(c) Moderate Wind

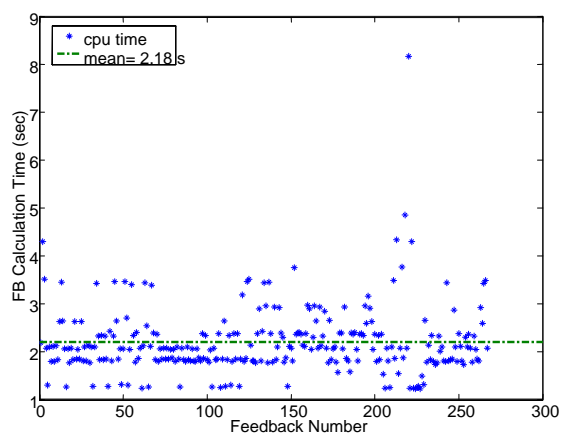

(e) Exaggerated Wind

Fig. 15 Feedback Computation Times for CL System

American Institute for Aeronautics and Astronautics 
For all wind disturbance cases, the average cpu computation time was $2.78 \mathrm{sec}$. As noted earlier, it is possible to reduce this time by a factor of 100 . Nonetheless, $2.78 \mathrm{sec}$ is quite adequate for declaring the computational speed as "real-time" as defined by the theory developed in Ref. 16 and validated by the numerical experiments reported in this paper.

\section{Conclusions}

In this paper, an optimal nonlinear feedback guidance law was constructed based on recent advances in pseudospectral methods. It was shown that real-time computation of the open-loop optimal controls can effectively be used for entry guidance in the presence of large uncertainties and disturbances that include hurricane-force wind gusts greater than Category 5 (Note: Hurricane Katrina was a Cat 4). Three significant advantages of this guidance scheme are: (1) that it does not require any advance knowledge of feedback computation times; hence, no prediction method is required; (2) tracking control is not required and as such the system is able to use the disturbances to its benefit, if any; and (3) provides the capability of recovering from failure and abort scenarios that would not be recoverable without rapid trajectory reshaping. Overall, this paper demonstrates the viability of this nonlinear feedback method for autonomous, on-board reentry applications.

\section{Acknowledgments}

The authors would like to extend their deep appreciation to Dr. Pooya Sekhavat, Guidance, Navigation, and Control (GNC) Laboratory, Naval Postgraduate School, for his help with implementing and debugging the feedback algorithm. This paper could not have been completed without his help.

\section{References}

${ }^{1}$ Harpold, J C., and Graves, C.A., "Shuttle Entry Guidance,” The Journal of Astronuatical Sciences, Vol. 27, No. 3, pp.239-268, Jul-Sep 1979.

${ }^{2}$ Oppenheimer, M.W., and Doman, D.B., "Reconfigurable Control Design for the X-40A with In-Flight Simulation Results," Proceedings of the 2005 AIAA Guidance, Navigation, and Control Conference, AIAA Paper No. 2004-5017, Aug 2004.

${ }^{3}$ Oppenheimer, M.W., and Doman, D.B., "Reconfigurable Inner Loop Control of a Space Maneuvering Vehicle," Proceedings of the 2003 AIAA Guidance, Navigation, and Control Conference, AIAA Paper No. 20035358, Aug 2003.

${ }^{4}$ Bolender, M.A., and Doman, D.B., “Nonlinear Control Allocation Using Piecewise Linear Functions,” Journal of Guidance, Control, and Dynamics, Vol.27, No. 6, pp.1017-1027, 2004.

${ }^{5}$ Doman, D.B., and Oppenheimer, M.W., "Integrated Adaptive Guidance and Control for Space Access Vehicles, Volume 1: Reconfigurable Control Law for X-40A Approach and Landing," AFRL IAG\&C Technical Report, Wright-Patterson AFB, OH, 2004.

${ }^{6}$ Schierman, J.D., Hull, J.R., and Ward, D.G., "On-Line Trajectory Command Reshaping for Reusable Launch Vehicles," Proceedings of the 2003 AIAA Guidance, Navigation, and Control Conference, AIAA Paper No. 20035439, Aug 2003.

${ }^{7}$ Vinh, Busemann, and Culp, Optimal Trajectories in Atmospheric Flight (Studies in Astronautics), Elsevier, Amsterdam, 1981.

${ }^{8}$ Regan, F.J., and Anandakrishnan, S.M., Dynamics of Atmospheric Re-Entry, AIAA Education Series, Washington D.C., 1993.

${ }^{9}$ Fahroo, F., Doman, D. and Ngo, A., “'Modeling Issues in Footprint Generation for Reusable Launch Vehicles," Proceedings of the 2003 IEEE Aerospace Conference, Big Sky, MT, Mar 2003.

${ }^{10}$ Fahroo, F., Doman, D. and Ngo, A., "'Footprint Generation for Reusable Launch Vehicles Using a Direct Pseudospectral Method," Proceedings of the American Control Conference, Denver, CO Jun 2003.

${ }^{11}$ Fahroo, F. and Doman, D., "A Direct Method for Approach and Landing Trajectory Reshaping with Failure Effect Estimation," Proceedings of the 2004 AIAA Guidance, Navigation, and Control Conference, AIAA Paper No. 2004-4772, Aug 2004. 
${ }^{12}$ Shaffer, P.J., Ross, I.M., Oppenheimer, M.W., Doman, D.B., “Optimal Trajectory Reconfiguration and Retargeting for a Reusable Launch Vehicle,” Proceedings of the 2005 AIAA Guidance, Navigation, and Control Conference, AIAA Paper No. 2005-4168, Aug 2005.

${ }^{13}$ Josselyn, S. and Ross, I.M., "Rapid Verification Method for the Trajectory Optimization of Reentry Vehicles," Journal of Guidance, Control, and Dynamics, Vol.26, No. 3, pp.505-508, 2002.

${ }^{14}$ Josselyn, S. and Ross, I.M., "Sensitivity Analysis for Rapid Prototyping of Re-Entry Vehicles," Proceedings of the 2003 AIAA Guidance, Navigation, and Control Conference, AIAA Paper No. 2003-5500, Aug 2003.

${ }^{15}$ Ross, I.M., Gong, Q., Fahroo, F., and Kang, W., "Practical Stabilization Through Real-Time Optimal Control,” Proceedings of the American Control Conference, Minneapolis, MN Jun 2006.

${ }^{16}$ Ross, I.M., Sekhavat, P., Gong, Q., and Fleming, A., "Pseudospectral Feedback Control: Foundations, Examples and Experimental Results," Proceedings of the 2006 AIAA Guidance, Navigation, and Control Conference, AIAA Paper No. 2004-6354, Aug 2006.

${ }^{17}$ Ross, I.M., and Fahroo, F., “Legendre Pseudospectral Approximations of Optimal Control Problems,” Lecture Notes in Control and Information Sciences, Vol. 295, Springer-Verlag, new York, pp.327-342, 2003.

${ }^{18}$ Ross, I.M., and Fahroo, F., "Pseudospectral Knotting Methods for Solving Optimal Control Problems,” Journal of Guidance, Control, and Dynamics, Vol. 27, No. 3, pp.397-405, 2004.

${ }^{19}$ Ross, I.M., Fahroo, F., and Gong, Q., “A Spectral Algorithm for Pseudospectral Methods in Optimal Control,” Proceedings of the $6^{\text {th }}$ IASTED International Conference on Intelligent Systems and Control, Honolulu, HI, 2004.

${ }^{20}$ Ross, I.M., and Fahroo, F., “User’s Manual for DIDO 2002: A MATLAB Application Package for Dynamic Optimization,” NPS Technical Report AA-02-002, Department of Aeronautics and Astronautics, Naval Postgraduate School, Monterey, CA, June 2002.

${ }^{21}$ Shaffer, Patrick J., Optimal Trajectory Reconfiguration and Retargeting for the X-33 Reusable Launch Vehicle, Thesis, Naval Postgraduate School, Monterey, CA, 2004.

${ }^{22}$ Phillips, S., “Earth's Atmosphere," NASA Exploration Database [online], URL: http://liftoff.msfc.nasa.gov/academy/space/atmosphere.html [updated 01 Dec 1995].

${ }^{23}$ Smith, C., "Monthly/Seasonal Climate Composites," NOAA-CIRES Climate Diagnostics Center [online database], URL: http://www.cdc.noaa.gov/cgi-bin/Composites/printpage.pl [updated 25 Oct 2005].

${ }^{24}$ Bluestein, Howard B., Synoptic-Dynamic Meteorology in Midlatitudes: Volume II: Observations and Theory of Weather Systems (Synoptic-Dynamic Meteorology in Midlatitudes), Oxford Univ. Press, New York, 1993.

${ }^{25}$ NOAA, "NCEP/NCAR Reanalysis Project at CDC," NOAA-CIRES Climate Diagnostics Center [online database], URL: http://www.cdc.noaa.gov/cdc/reanalysis/ [updated 30 May 2003].

${ }^{26}$ NOAA/NWS, “The Saffir-Simpson Hurricane Scale,” National Hurricane Center - Tropical Prediction Center [online database], URL: http://www.nhc.noaa.gov/aboutsshs.shtml [updated 22 Jun 2006].

${ }^{27}$ Ross, I.M., and Fahroo, F., "Issues in the real-time computation of optimal control," Mathematical and Computer Modeling, An International Journal, Elsevier, Vol. 43, pp.1172-1188, 2006.

${ }^{28}$ Ross, I.M., Gong, Q., and Sekhavat, P., "A Simple Technique for Low-Thrust High-Accuracy Trajectory Optimization,” $16^{\text {th }}$ AAS/AIAA Space Flight Mechanics Conference, Tampa, FL, Paper No. AAS 06-150, Jan 2006. 